Prospect. Univ. (2010)

\title{
DISEÑO DE EQUIPOS E INSTRUMENTOS PARA LA COLECCIÓN Y CONSERVACIÓN DE SEMEN EN ALPACAS (Vicugna pacos) ${ }^{22}$
}

Espinoza Molina, Saúl23, Pantoja Esquivel, Rafael A. ${ }^{24}$

Facultad de Zootecnia de la Universidad Nacional del Centro del Perú

\section{RESUMEN}

El trabajo de investigación se realizó en la etapa de laboratorio en la Granja Agropecuaria de Yauris de la Universidad Nacional del Centro del Perú, situada a 3316 m.s.n.m., a 02 Km de la ciudad de Huancayo, ubicada en Distrito de El Tambo, provincia de Huancayo, Departamento de Junín. y la etapa de campo en la Empresa Comunal Santo Domingo de Cachi, del distrito de Yanacancha de la provincia de Concepción del departamento de Junín. Entre la problemática de la reproducción asistida en alpacas está la colección óptima, representativa y repetitiva del eyaculado ¿Existirán equipos e instrumentos adecuados para la colección de semen en alpacas para su conservación y posterior utilización por los sistemas de reproducción como la inseminación artificial? ; La hipótesis planteada fue: Existen equipos e instrumentos adecuados a las características sui géneris de cópula de la alpaca para obtener semen de calidad y cantidad, se planteó el siguiente objetivo general: Diseñar equipos e instrumentos para la colección de semen en alpacas machos para su posterior utilización en programas de inseminación artificial. Como objetivos específicos: a) Diseñar un electroeyaculador (EE) para colección de semen en alpacas, b) Diseñar una frazadilla eléctrica para la colección de semen por vagina artificial (VATe), c) Evaluar algunas características seminales por ambos métodos de colección de semen. Los resultados hallados fueron los siguientes: Las características del semen obtenidos son afectadas por el método de colección empleado. Se obtuvo semen de la alpaca macho por el método de colección con vagina artificial termoeléctrica cubierta con frazadilla eléctrica en forma parcial, no todos los machos responden a éste método de colección de semen. Se colectó semen del jaiñu por electroeyaculación previo cambio de corral, estimulación de la orina y administración de Xilazina Clorhidrato (sedante) 0,25 - 1,5 $\mathrm{ML} / 100 \mathrm{Kg}$ de p.v. La colección de semen con VATe se realizó en 04 machos. Se colectaron 16 muestras de semen y se obtuvieron los siguientes resultados: el volumen promedio fue de 1,6 $\pm 0,8 \mathrm{ml}$, encontrándose valores de 0,2 a $2 \mathrm{ml}$; el pH promedio fue de 7,5 \pm 0,2 , con valores extremos de 7,2 y 8,0 . La motilidad se caracterizó por un movimiento oscilatorio, sin motilidad masal Para la colección de semen con electro eyaculador (EE), el volumen promedio fue de 2,73 $\pm 0,8 \mathrm{ml}$, con valores que varían de 0,2 a $3,3 \mathrm{ml}$; el $\mathrm{pH}$ promedio fue de $7,2 \pm 0,21$, con valores que varían entre 7,0 y 8,0 ; la vitalidad promedio fue de $72,3 \pm 21,9 \%$, con valores que fluctuaron entre $0 \%$ y $80 \%$; la motilidad tipo 0 varió ente 20 y 100 , alcanzando un promedio de $60,5 \pm 23,4 \%$; el promedio de motilidad tipo1 fue $21,7 \pm 15,7 \%$, con valores que variaron entre $0 \%$ y $62 \%$, el promedio para la motilidad de tipo 2 fue de $10,4 \pm 11,9 \%$, con valores extremos de 0 y $64,2 \%$. El promedio para la vitalidad tipo 3 resultó de $7,4 \pm 9,3 \%$, con valores extremos de 0 y 31,7 , requiere ser validado.

Palabras clave: inseminación artificial, colección de semen, alpacas

\section{DESIGN OF EQUIPMENT AND INSTRUMENTS FOR THE COLLECTION AND PRESERVATION OF SEMEN IN ALPACA (Vicugna pacos) \\ ABSTRACT}

The research was conducted in the laboratory stage on the Granja Agropecuaria de Yauris of the Universidad Nacional del Centro del Peru, located at $3316 \mathrm{mosl}, 2 \mathrm{~km}$ from Huancayo city, located in El Tambo district, Huancayo province, department of Junín. and field stage in the Communal Enterprise Santo Domingo de Cachi, Yanacancha district of the department of Junin. Among the issues of assisted reproduction in animals is the best collection, representative and repetitive of the ejaculate. Are there adequate equipments and instruments for the collection of sperm in alpacas for conservating and subsequent use of artificial systems such as artificial insemination? The stated hypothesis was: There are adequate equipments and instruments to the sui generis nature of the copula for alpaca semen quality and quantity. General objetive: Design equipments and instruments for the collection of semen in male alpacas for subsequent use in artificial insemination programs. Specific purpose: a) Design an electroeyaculador (EE) for semen collection in alpacas, b) Design an electrical frazadilla for semen collection by artificial vagina (Vate) c) evaluate some characteristics of both seminal collection methods of semen. The findings were as follows: The characteristics of semen obtained are affected by the method of collection employed. We collected semen from the male alpaca by the method of collection by artificial vagina covered with thermoelectric frazadilla in part, not all males responded to this method of collection of semen. Jaiñu semen was collected by hand after electroeyaculación poultry, and stimulation of urine administration of Xylazine hydrochloride (sedative) $0.25-1.5 \mathrm{ml} / 100 \mathrm{~kg} \mathrm{pv}$ The collection of semen with Vate was performed in 04 males. Collected 16 samples of semen and the following results: the average volume was $1.6 \pm 0.8 \mathrm{ml}$, with values of 0.2 to $2 \mathrm{ml}$, the average $\mathrm{pH}$ was $7.5 \pm 0.2$, with extreme values of 7.2 and 8.0 . Motility was characterized by an oscillatory movement without masal motility for semen collection with electro ejaculated (EE), the average volume was $2.73 \pm 0.8 \mathrm{ml}$, with values ranging from 0.2 to $33 \mathrm{ml}$, the average $\mathrm{pH}$ was $7.2 \pm 0.21$, with values ranging between 7.0 and 8.0 , the average life was $72.3 \pm 21.9 \%$, with values ranging from $0 \%$ and $80 \%$ motility rate 0 ranged between 20 and 100 , reaching an average of $60.5 \pm 23.4 \%$; type 1 the average motility was $21.7 \pm 15.7 \%$, with values ranging between $0 \%$ and $62 \%$, the average motility rate 2

\footnotetext{
${ }^{22}$ Trabajo de investigación fue recibido el 07/05/2010 retornado para su revisión el 15/06/2010 y aprobado para su publicación 16/11/2010 Departamento Académico De Producción Animal Y Gestión Ambiental, Programa Biotecnología, UNCP.

23 surysaul@hotmail.com

24 centrozoot@hotmail.com
} 
was $10.4 \pm 11.9 \%$, with extreme values of 0 and $64.2 \%$. The average for the vitality of type 3 was $7.4 \pm 9.3 \%$, with extreme values of 0 and 31.7 , must be validated.

Key words: Artificial insemination, semen collection, alpaca

\section{INTRODUCCIÓN}

Las alpacas constituyen el mayor aporte de la cultura incaica y preincaica a la ganadería peruana y mundial. De las cuatro especies de camélidos sudamericanos, la alpaca (Vicugna pacos) es el de mayor importancia económica y social, por ser domesticada y producir fibra fina $y$ carne de excelentes cualidades bromatológicas y bien cotizada económicamente en el mercado internacional y local cuya aceptación va en aumento especialmente el producto carne. Sin embargo, el desarrollo tecnológico de su crianza y producción es pobre debido al insuficiente conocimiento de su biología y de la realidad socioeconómica en el que esta actividad se desarrolla. Entre los aspectos reproductivos más importantes y menos estudiados se encuentra el semen y sus características: conocerlas es indispensable para poder introducir en la ganadería andina técnicas de propagación y mejoramiento de germoplasma existentes para la aplicación de biotecnologías reproductivas como la inseminación artificial, fertilización in vitro, etc. que permitan mejorar la producción y productividad actual de su especialidad productiva, y por lo tanto el nivel de vida del poblador andino que lo cuida y depende de esta actividad productiva.

La inseminación artificial como la transferencia de embriones son biotecnologías reproductivas que han contribuido al avance o progreso genético de la mayoría de las especies animales de mayor interés zootécnico y comercial; sin embargo, en los camélidos sudamericanos como la alpaca, el avance en la implementación de estos proceso reproductivos artificiales es poco pese al esfuerzo dedicado por muchos investigadores peruanos, por existir algunas limitaciones de orden biológico suí géneris de la alpaca y que requieren diseñar equipos e instrumentos específicos, como la colección y recolección de semen, conservación de semen al medio ambiente, refrigerado y si es posible el congelamiento que viabilicen estas biotecnologías reproductivas. En ese sentido el objetivo general fue el de diseñar equipos e instrumentos para la colección de semen en alpacas machos para su posterior utilización en programas de inseminación artificial, dentro del cual se planteó también, diseñar un electroeyaculador (EE) para colección de semen en alpacas, diseñar una frazadilla eléctrica para la colección de semen por vagina artificial (VATe) y evaluar algunas características seminales por ambos métodos de colección de semen.

\section{MATERIALES Y MÉTODOS}

\subsection{LUGAR Y FECHA}

El trabajo de investigación se realizó, en su etapa inicial en laboratorio, y de campo en la Granja Agropecuaria de Yauris de la Facultad de Zootecnia de la Universidad Nacional del Centro del Perú, situada a 3200 m.s.n.m., al oeste y a $02 \mathrm{Km}$ de la ciudad de Huancayo.

La Evaluación de la construcción y funcionamiento de los equipos e instrumentos para colección de semen en la alpaca se realizó entre los años 2008 a I 2009.

- Etapa de Campo. Se ha procedido a realizar la recolección de información biométrica de los principales órganos del aparato reproductor del jaiñu o alpaca macho, órganos comprometidos con el diseño de equipos e instrumentos para la colección de semen.

- Etapa Inicial de Gabinete. Se ha procedido a realizar un revisión bibliográfica sobre el comportamiento reproductivo de la las alpacas machos "jaiñus" para considerar en el diseño de instrumentos y equipos iniciales de construcción como por ejemplo la vagina artificial, el maniquí y el lectroctroeyaculador bipolar con fuente de energía propia.

\subsection{Metodología}

\section{De las Alpacas Machos "Jaiñus"}

En la Granja Agropecuaria de Yauris se dispone de tres alpacas machos un adulto (de 06 años de edad), dos jóvenes de (03 años de edad) y dos hembras con los cuales se acondicionó y se hicieron las primeras pruebas de colección de semen por los métodos de la vagina artificial y electroeyaculación.

En la segunda etapa de campo se dispuso de 10 machos reproductores de la Empresa Comunal Santo Domingo de Cachi del distrito de Yanachacha y provincia de Concepción, del que se seleccionarán del rebaño de alpacas del sexo macho que hayan tenido antecedentes de haber servido en el empadre de la campaña anterior, a los que se complementaron con la inspección visual y palpación de genitales externos y provengan genéticamente de clase alta Stud o Súper" 0 de plantel con registro genealógico conducido por el 
CONACS y la empresa comunal. Además, se contó con otras dos hembras en franco celo para la colección de semen por vagina artificial termoeléctrica.

\section{De los materiales y equipos:}

Para el presente estudio se utilizaron: Maniquí de madera en posición decúbito ventral, electroeyaculador, vagina artificial termoeléctrica, termos, tetera, fuente de energía eléctrica, fundas de látex rectas, preservativos de uso humano, gomas de unión, glicerina líquida, mallas de alambre, vaqueta de vidrio, microscópio simple de espejos, platina caldeable, porta y cubre objetos, xilazina clorhidratado (sedante), jeringas y agujas hipodérmicas, alcohol, algodón y gasa.

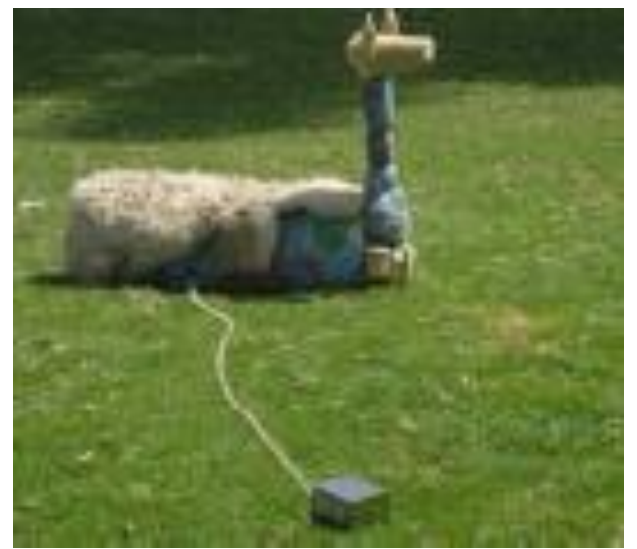

Figura 1.- Maniquí

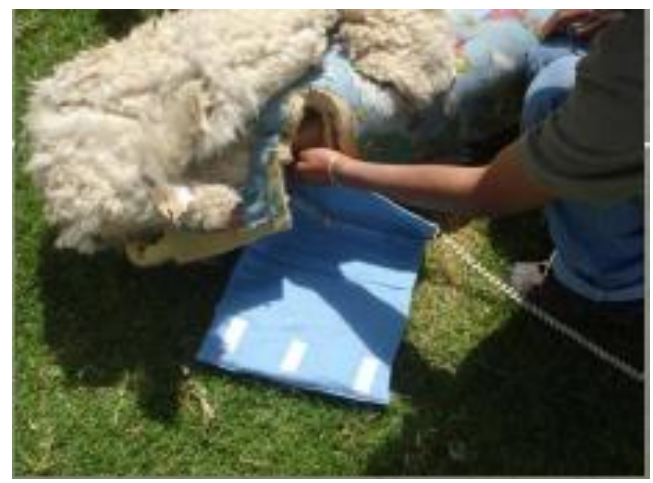

Figura 2.- Vagina artificial termoeléctrica

\subsection{Evaluación de las Muestras de Semen Volumen}

El volumen de semen se midió directamente en los tubos de colección graduados. El resultado se expresará en mililitros (ml).

\section{$\mathrm{pH}$}

Se midió con cinta de papel indicador de $\mathrm{pH}$ con rango de 6,0 a 9,0 (pHydrion Microfine).

\section{Vitalidad (Porcentaje de vivos y muertos)}

De la muestra debidamente homogenizada se tomarán $10 \mathrm{uL}$ con un micro pipeta automática graduada ( de 5$40 \mathrm{uL}$, con escala de 0,5 uL,Labsystems) colocándolos sobre una lámina porta objetos. Sobre la gota de semen se colocará $5 \mathrm{uL}$ del colorante Eosina- $Y$ al $0,5 \%(\mathrm{p} / \mathrm{v})$, se homogeniza y se cubre luego con una lámina cubre-objetos. Después de 1 a 2 minutos se observará el preparado a $400 \mathrm{X}$ de aumento con luz intensa. Se contarán los espermatozoides en tres campos de microscopio por muestra; se considerarán vivos a los espermatozoides no teñidos, y muertos a los teñidos. El resultado se expresará en porcentaje de espermatozoides vivos.

\section{Motilidad}

Se colocó una gota de (10 a 15uL) de semen homogenizado sobre una lámina portaobjetos y se cubrió con una lámina cubreobjetos de $24 \times 24 \mathrm{~mm}$. El preparado se dejó reposar por un minuto. Se observará con 400X. Se evaluaron por lo menos dos campos del microscopio por muestra. El resultado se expresará en porcentaje de espermatozoides según los tipos de motilidad descritos a continuación:

$-0=$ Sin motilidad.

$-1=$ Motilidad no progresiva (in situ)

$-2=$ Motilidad lineal lento o no lineal, perezoso.

- $3=$ Motilidad progresiva rápida y lineal.

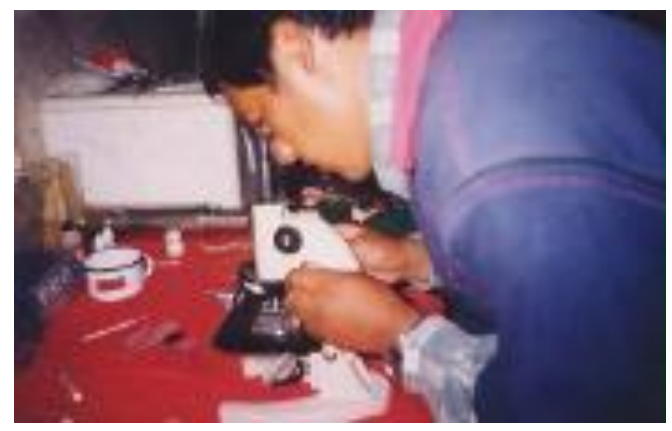

\section{DEL ELECTROEYACULADOR}

A continuación se describirá los materiales empleados y la elaboración por etapas, paso a paso del transductor, uno de los objetivos que se persigue en este proceso, es que este instrumento puede ser elaborado por aquellos que tengan interés en su utilización, ya que requiere un mínimo de herramientas 
e insumos de uso cotidiano y que fácilmente se pueden obtener.

\section{METODOLOGÍA EMPLEADA}

\section{ETAPA I:}

Se procedió a diseñar el transductor que servirá como electrodo, para ello se consideró las dimensiones anatómicas del sistema excretor de una alpaca adulta, en donde se evaluó que el recto donde se introducirá el electrodo tiene una longitud entre 20 a $25 \mathrm{~cm}$, con un diámetro máximo de $3,5 \mathrm{~cm}$. Entonces nuestro electrodo debe tener una dimensión entre los $20 \mathrm{~cm} x$ $2,5 \mathrm{~cm}$ como máximo.

Para ello se utilizó como base del electrodo un tubo de pvc de $18 \mathrm{~cm}$ cuyo diámetro es de $2 \mathrm{~cm}$ al cual se le enrolló el alambre de cobre $\mathrm{N}^{0} 10$ arrollado en forma de espiral sobre todo el tubo tanto para el polo negativo y para el positivo. Luego de ello se rellenó con base de fibra de vidrio llegando a un diámetro total de $2,5 \mathrm{~cm}$ tal como se observa en la figura.
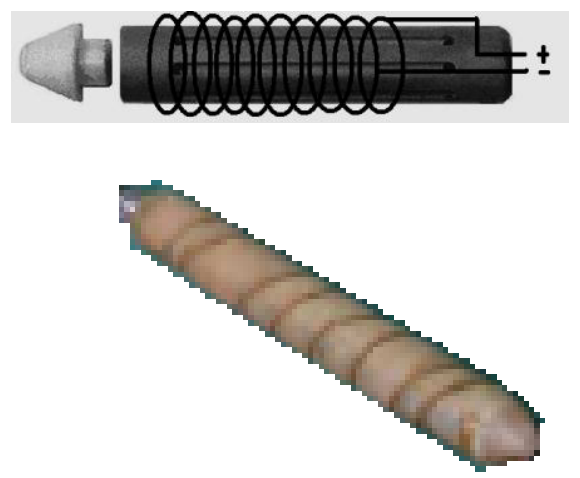

\section{2a. Etapa}

El diagrama esquemático que se muestra en la figura consiste en un circuito inversor común, construido a partir de compuertas de circuito integrado CMOS (IC1) 4093 que entregan la señal generada a un transistor de efecto de campo de compuerta aislada (Q1) IRF630.

La primera compuerta del IC1 funciona como un oscilador, cuya frecuencia y ciclo de actividad puede ser ajustada por medio de potenciómetro (P1) quien da la intensidad de voltaje y por potenciómetro (P2) quien modifica la frecuencia de los pulsos.

Resistencias: $\quad \mathrm{R} 1=10 \mathrm{Kohms}, \mathrm{R} 2=1 \mathrm{~K}$ ohm, R3= 1 Mohm, R4=5,6 Kohm, R5 560 ohms, Circuitos Integrados: $\mathrm{ICI}=\mathrm{CD}$ 4091, Transistor: IFR630,
Capacitores: C1 $=0,47$ PF, C2 $=100$ UF $/ 25$ Voltios, Potenciometros: P1 $=10 \mathrm{Kohms}, \mathrm{P} 2=100 \mathrm{Kohms}$ cada uno de 2 wats, D1, D2 = LEDS, Interruptor para 10 amp, transformador 220v / 12 v 1 amp (modificado).

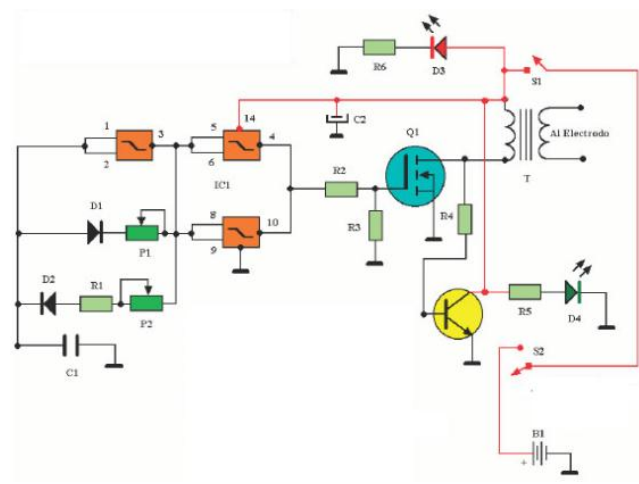

Las otras dos compuertas del IC1, conectadas en paralelo, funcionan como "separadoras" e inversoras que entregan la señal de estímulo a la base de Q1.

Este transistor opera en las zonas de corte y saturación, con lo cual en la salida de este componente se tiene un pulso de alta corriente que puede excitar al transformador (TI) que, por tener una relación de transformación elevada, entrega en el secundario, pulsos de alta tensión y baja corriente, la aparición de estos pulsos en el colector de Q1, a través de la resistencia R4 excitan la base del transistor Q2 y el LED de color verde enciende dependiendo de la intensidad y frecuencia, haciendo las veces de piloto para monitorear la salida de energía hacia el transductor que excitará al animal. El LED D3 de color rojo funciona como piloto de encendido.

El transformador T1 que se empleó se tuvo que reconstruir modificando el arrollamiento primario de 1,746 espiras con alambre esmaltado número 36 y arrollamiento secundario de 275 espiras con alambre esmaltado número 28 , con esto tendremos una relación de 55 voltios/12voltios.

Todo el sistema del circuito se ensambló en una placa de circuito impreso diseñado para colocar todas las piezas electrónicas.

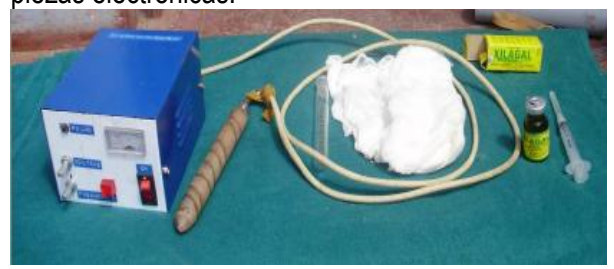




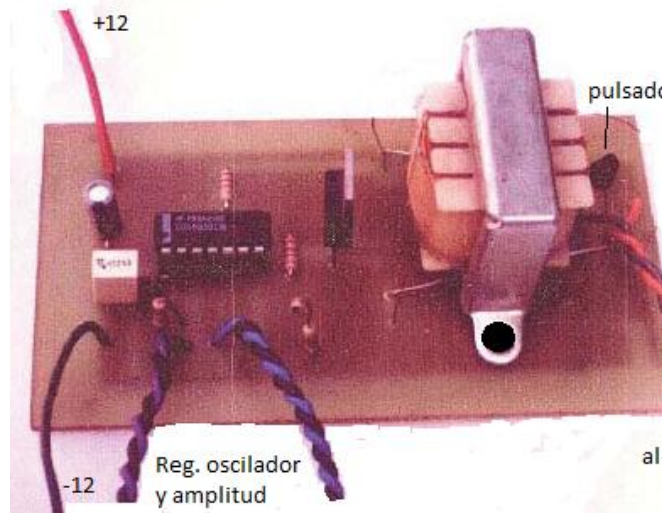

\subsection{Frazadilla Termoeléctrica}

Para su diseño y elaboración se inició en base a un cálculo sencillo de resistencia y conductividad eléctrica según la resistencia que ofrece un material al paso de la corriente eléctrica y se utilizó la siguiente fórmula

$$
R=\rho \frac{\mathrm{I}}{\mathrm{s}}
$$

De donde:

$\mathrm{R}=$ Resistencia del material en ohm ( $\Omega$ ), en este caso del alambre de nicrom No39

$P=$ coeficiente de resistividad o resistencia específica del material en

$\Omega \cdot \mathrm{mm}^{2}$

m , a una temperatura dada, para el nicrom fue de $1,5 \Omega \cdot \mathrm{mm}^{2}$ I

m

I = Longitud del alambre de nicrom en metros.

$\mathbf{s}=$ Superficie 0 área transversal del material en $\mathrm{mm}^{2}$.

Y para determinar la potencia en energía calórica que se necesita esta fórmula se relación con la de potencia: Potencia en wats $=$ Intensidad $(\mathrm{amp}) \times \mathrm{E}$ (voltios).

Ente otros materiales se tiene, franela, fibra de vidrio, esponja, sujetadores pega pega.

\subsection{Del Análisis Estadístico:}

Se estiman los parámetros estadísticos media poblacional (u), desviación estándar poblacional (s), rango $(R)$ y coeficiente de variabilidad $(C V)$, para los resultados totales de los dos métodos de colección ensayados.

Utilizando los datos transformados y el macho como unidad experimental, se comparan los promedios de $\mathrm{pH}$ y volumen de las muestras de semen colectadas por vagina artificial termoeléctrica y por electroeyaculador, por medio de la prueba de "t" de Student (Calzada, 1968).
En las muestras obtenidas por "electroeyacuación" se estimaron la variabilidad intraindividual e interindividual. Las diferencias entre las muestras de semen de un mismo macho se discuten a partir de su promedio y coeficiente de variabilidad.

\section{RESULTADOS}

\subsection{Para los Métodos de Colección de Semen}

\subsubsection{De Vagina Artificial termoeléctrica (VATe)}

Se pudo diseñar una frazadilla eléctrica, La cual tuvo una dimensión de $40 \mathrm{~cm} \times 30 \mathrm{~cm}$, dentro del cual se insertó el alambre de nicrom con una longitud de 5 metros y con un voltaje de 12 voltios se obtuvo una potencia de 25 wats, necesarios para mantener la frazadilla a una temperatura de $37^{\circ} \mathrm{C}$ a $38^{\circ} \mathrm{C}$, la cual posteriormente sirvió para envolver la vagina artificial permitiendo mantener la temperatura del semen a los $36^{\circ} \mathrm{C}$ como promedio.

La colección de semen con VATe se realizó en 04 machos. Se colectaron 16 muestras de semen y se obtuvieron los siguientes resultados: el volumen promedio fue de 1,6 $\pm 0,8 \mathrm{ml}$, encontrándose valores de e 0,2 a $2 \mathrm{ml}$.

\subsubsection{Electroeyaculación, pH y motilidad}

Se colectaron 27 muestras de semen de 09 alpacas machos de la raza Huacaya, 14 de éstas fueron colectadas en 03 machos (EE1,EE2 y EE3), Las 14 muestras restantes se colectaron de 06 machos ( EE4,EE5,EE6,EE7,EE8 y EE9), de éstos 06 machos, 03 son reproductores que venía de dar servicios e un grupo de hembras durante una semana y 03 de plantel, estos machos son seleccionados por la excelencia de su fenotipo (buen tamaño, fibra fina) y son entrenados para ser presentados en exposiciones ganaderas. Se obtuvieron los siguientes valores para las características del semen de alpacas machos: Volumen promedio de $2,73 \pm 0,8 \mathrm{ml}$, con valores que varían de 0,2 a $3,3 \mathrm{ml}$; el $\mathrm{pH}$ promedio fue de 7,2 \pm 0,21 , con valores que varían entre 7,0 y 8,0 ; la vitalidad promedio fue de $72,3 \pm 21,9 \%$, con valores que fluctuaron entre $0 \%$ y $80 \%$; la motilidad tipo 0 varió ente 20 y 100 , alcanzando un promedio de 60,5 $\pm 23,4 \%$; el promedio de motilidad tipo1 fue $21,7 \pm$ $15,7 \%$, con valores que variaron entre $0 \%$ y $62 \%$, el promedio para la motilidad de tipo 2 fue de 10,4 \pm $11,9 \%$, con valores extremos de 0 y $64,2 \%$. El promedio para la vitalidad tipo 3 resultó de $7,4 \pm$ $9,3 \%$, con valores extremos de 0 y 31,7 ; requiere ser validado 


\subsubsection{De la Evaluación de las Muestras de Semen} Las muestras de semen obtenidos directamente en los tubos de colección graduados por los dos métodos de colección fueron depositados en baño maría a una temperatura de $37^{\circ} \mathrm{C}$, para el examen del semen; donde se evaluaron las siguientes características: Volumen, color, $\mathrm{pH}$, vitalidad (porcentaje de vivos y muertos) y motilidad.

\section{DISCUSIÓN}

\subsection{De Vagina Artificial termoeléctrica (VATe)}

Este método ha sido ensayado y utilizado en alpacas por Sumar y Leyva (1981), Leyva y col. (1984) y Chipana y col (1990). Se colectaron el semen de alpacas machos con VATe; se usó una modelo para ovinos construida de un tubo de plástico o PVC, con válvula para regular la presión y fundas de látex, el que fue recubierto con una frazadilla termoeléctrica; al que se acopló al maniquí en posición (decúbito ventral). Machos y hembras se mantuvieron separados; momentos antes de la colección se juntaron en un corral a una hembra receptiva y a un macho para el entrenamiento. Se trabajó con diez machos de la E.C. Santo Domingo de Cachi; de los cuales sólo respondieron al entrenamiento 04 machos: (VATe1, VATe2,VATe3 y VATe4), a los que se le provocó una monta natural en el maniquí que previamente tenía adosada la vagina artificial termoeléctrica atemperada con agua tibia a $38^{\circ} \mathrm{C}$, por el tiempo de cópula. Se tomó diferentes números de muestras de cada uno de ellos.

\subsection{De la Electroeyacuación (EE)}

En este estudio se ha aplicado el método de colección de semen según lo describe Férnández Baca y Calderón, (1966); Calderón y col. (1968), con algunas modificaciones, como el uso de tranquilizantes administrados a los donadores de semen. Se colectó semen de jaiñus que previamente se cambiaron de corrales de reposo para estimular la orina como característica de demarcar su territorio y administrando en seguida un tranquilizante (Xilazina clorhidrato), que permitió manipular al animal y sujetar el pene para la colección directa del semen en el tubo de colección entibiado y protegido con gasa. De esta manera se obtuvieron diferente número de muestras de semen de 09 alpacas de la empresa comunal Santo Domingo de Cachi. (EE1,EE2,EE3,EE4,EE5,EE6,EE7,EE8 Y EE9).

\subsection{Motilidad}

La motilidad, se caracterizó por un movimiento oscilatorio, sin motilidad masal, dichas características concuerdan con la escrita por Novoa, C (1991).

\section{CONCLUSIONES}

\section{De la Construcción de equipos e instrumentos para colección de semen en alpacas. \\ - Se logró confeccionar un electro eyaculador (EE) con especificaciones técnicas viables para colección de semen en alpacas. \\ - Se logró construir una frazadilla eléctrica para la colección de semen con vagina artificial termoeléctrica atemperada (VATe).}

\section{De los Métodos de Colección de Semen.}

- Las características del semen obtenidos son afectadas por el método de colección empleado.

- Es posible obtener semen de la alpaca macho por el método de colección con vagina artificial termoeléctrica en forma parcial, pero no todos los machos responden a éste método de colección de semen.

- Es posible colectar semen del jaiñu por electroeyaculación previo cambio de corral, estimulación de la orina y administración de Xilazina Clorhidrato (sedante) $0,25-1,5 \mathrm{~mL} / 100 \mathrm{Kg}$ de p.v.

\section{Respecto a las Características del Semen. \\ El semen de alpaca colectado con vagina artificial termoeléctrica (VATe) presenta las siguientes características:}

La colección de semen con VATe se realizó en 04 machos. Se colectaron 16 muestras de semen y se obtuvieron los siguientes resultados: el volumen promedio fue de 1,6 $\pm 0,8 \mathrm{ml}$, encontrándose valores de 0,2 a $2 \mathrm{ml}$; el pH promedio fue de $7,5 \pm 0,2$, con valores extremos de 7,2 y 8,0 . La motilidad, se caracterizó por un movimiento oscilatorio, sin motilidad masal.

\section{REFERENCIAS BIBLIOGRÁFICAS}

- $\quad$ CALDERÓN, W; SUMAR, J.; FRANCO, E.. Avances en la Inseminación Artificial de las Alpacas (Lama pacos). Revista de la facultad de Medicina Veterinaria. UNMSM. 22:19-35. 1968.

- $\quad$ CHIPANA,0; ALVARADO,E.;CARDENAS,H. Colección y Dilución del Semen Alpacas. P.69. En: 13 Reunión Científica Anual (APPA). Libro de Resúmenes. Ayacucho, UNSCH. 97 p. 1990 
- $\quad$ Fernandez-BACA, S.; NOVOA, C. Primer Ensayo de inseminación Arificial de Alpacas (Lama pacos) con Semen de Vicuñas (Vicugna vicugna). Revisa de la Faculta d de Medicina Veterinaria UNMSM. 22: 9-18. 1968.

- $\quad$ NOVOA, C. Reproducción. Simposio: producción de Llamas y Alpacas. En: 12. Reunión Científica Anual de APPA. UNMSM. Lima. 1989.

- $\quad$ NOVOA, C; y LEYVA, V. Reproducción en Alpacas y Llamas. E.E. Marangani- La Raya, IVITA, FMV, Univ. San Marcos.. Publ. Cient. IVITA No26. 32 pgs. 1994.

- SAN MARTIN, M. Fisiología de la Reproducción de la Alpaca. An. Symp. Sobre Problemas Ganaderos. Lima. Perú. Pp 113-121. 1961.

- $\quad$ SUMAR,J. Fisiología de la Reproducción del Macho y Manejo Reproductivo. Avances y Perspectivas del Conocimiento de los Camélidos Sudamericanos. Oficina regional de la FAO. Santiago de Chile. 1991.

- SUMAR, J. Llamas y Alpacas. Reproducción e Inseminación Artificial en Animales. E.S.E. Hafez. Capítulo 15, 224-242 pp. Séptima Edición Mc GrawHill Interamericana. 2002.

- Foros de Electrónica, Comunidad Internacional de Electrónicos Powered by phpBB @ 2001,2005 phpBB Group. 2005. 